\title{
DC-DC 变换器的典型故障分类方法研究
}

\author{
徐㻇 ${ }^{1}$, 张一童 ${ }^{1}$, 潘震 ${ }^{2}$, 池程芝 ${ }^{2}$, 刘晓斌 ${ }^{3}$
}

$\left(\begin{array}{l}\text { 1.西北工业大学 电子信息学院, 陕西 西安 } 710129 ; \\ \text { 2.中国航空无线电电子研究所 航空电子系统综合技术重点实验室, 上海 } 200233 \\ \text { 3.西安现代控制技术研究所, 陕西 西安 } 710065\end{array}\right)$

\begin{abstract}
摘 要:DC-DC 变换器是综合模块化航电的电源转换模块的核心部件, 对其进行状态监测以及故障 诊断可以有效提高设备的可靠性,减少维修保障费用,极大地提高飞机的使用效能。基于 PSPICE 仿 真软件, 采用 Sepic 拓扑结构, 设计了 DC-DC 变换器模型, 并对 DC-DC 变换器的失效规律进行了分 析; 对 DC-DC 变换器的典型故障类型进行故障模拟,通过仿真获取相应的原始数据; 采用数据预处 理、特征提取与选择、多模型融合的处理框架对 DC-DC 变换器进行故障诊断分析。仿真验证了方法 的有效性。
\end{abstract}

\section{关 键 词:DC-DC 变换器; 特征提取; 故障分类; 多模型融合 \\ 中图分类号:TG156 文献标志码: A \\ 文章编号: 1000-2758(2021) 01-0071-06}

随着信息技术的推动和信息条件下作战需求的 牵引, 综合模块化航空电子系统的作用和地位日益 突出, 它是现代作战飞机完成导航与识别、控制与管 理等任务的先进综合集成平台。以 F-35 为代表的 新一代战机, 拥有先进的模块化航空电子系统, 实现 了机载资源的高度共享、分层系统的综合管理, 极大 地提升了战机的作战效能, 因此, 航空电子系统的可 靠性显得尤为重要 ${ }^{[1-3]}$ 。

作战飞机不可避免地装载了大量的用电设备, 综合模块化航电的电源转换模块 (power conversion module, PCM), 将飞机电源系统供电转换为内部机 架式电源的直流输人电压, 进而通过电源开关给各 通用功能模块供电。 PCM 模块是其他各通用功能 模块工作的基础, 其工作状态对其他通用功能模块 能否正常工作有着重要的影响。PCM 的核心部分 为 DC-DC 变换器, 其健康状况能够反映整个 PCM 的工作状态, 对其进行状态监测和故障诊断具有重 要的意义。文献 [4-5]对升压型变换器故障诊断方 法进行了研究。文献 $[6]$ 提出了零电压开关直流变 换器开关的短路故障和开路故障诊断方案。文献
[7-8]研究了三电平变换器的故障诊断方法。

传统的故障诊断与维修方法, 存在维修效率低、 维修成本高、可靠性差等问题。因此, 利用对 DCDC 变换器在线采集数据进行分析, 建立故障诊断 模型, 及时的发现故障, 采取有效管理措施, 具有重 要意义,可极大地提高飞机系统可靠性。

\section{DC-DC 变换器失效模式分析}

电力电子线路的性能退化通常是由电路中元器 件的失效引起。DC-DC 转换器的故障主要由一小 部分组件导致的。DC-DC 变换器失效概率较大的 部件主要有电解电容器、开关晶体管、电感和二极 管, 由于主电路工作频率较低, 不会对电感器件造成 大的损坏, 故在此不予考虑, 本文主要针对电解电容 器、开关晶体管和二极管提取故障特征参数, 建立故 障分类诊断模型。

铝电解电容即滤波网络中的滤波电容, 在 DCDC 变换器中主要用于滤波网络, 用来吸收电压纹 波进而达到平滑输出电压的作用, 所以铝电解电容 
的劣化会使 DC-DC 变换器输出的交流成分增加。 电解电容因具有成本低、容量大等优点被广泛应用 于滤波和电能的存储变换, 但其发生故障的概率很 大, 对输出电压有较大影响, 其性能的好坏直接决定 DC-DC 变换器的可靠性。

开关晶体管作为电力电子电路的功率转换器 件, 开关晶体管的故障将直接影响 DC-DC 变换器的 失效率、温升指标和技术性能。考虑到航空用途 PCM 的实际情况，一般采用 MOSFET 晶体管， MOSFET 晶体管可在高频率环境下作业。

功率二极管作为一种常用的功率器件, 在电力 电子电路中起着续流、整流等重要作用。肖特基二 极管作为一种常用的功率二极管, 在 DC-DC 变换器 中也较为常见, 具有低电压、大电流以及速度快等优 点, 但容易在过大的反向电压和电流作用下, 出现反 向击穿的现象。

本文在 PSPICE 环境下设计了典型的基于 Sepic 拓扑结构的 DC-DC 变换器, Sepic 型变换器是 DC-DC 变换器的一种, 它允许输出电压大于、小于 或等于输人电压的变换器, 由主控开关控制, 且通过 主回路上的电容能够实现输出和输人的隔离, 广泛 应用于航空领域。通过设置元器件参数可以实现预 期的升降压变换。PSPICE 基于 FORTRAN 语言, 是 由 SPICE 发展而来的通用电路分析软件, 主要用于 大规模集成电路的计算机辅助设计, 具有丰富的元 器件库, 是最流行的电力电子线路 $\mathrm{EDA}$ 软件之一。 PSPICE 不仅可进行数模混合电路的仿真, 而且具有 PSPICE-MATLAB 接口软件模块来用于测试实际电 子设计的系统级接口, 便于我们后续对仿真结果进 行数据交互与分析。

搭建的 PSPICE 模型图 1 所示。在 PSPICE 中 可以对不同类型的故障进行仿真, 采集故障数据。 本文共设置了 5 种故障模式: MOS 管短路故障、二 极管开路故障、二极管短路故障、电解电容击穿故障 和电解电容开路故障。在 PSPICE 中对短路故障的 模拟方法为: 为短路故障元件并联一个 SW_open 开 关, 设置开关在 $6 \mathrm{~ms}$ 时刻由断开状态切换至闭合状 态, 模拟元器件的突然短路故障。开路故障的模拟 方法为: 为开路故障元件串联一个 $\mathrm{SW}_{-}$close 开关, 设置开关在 $6 \mathrm{~ms}$ 时刻由闭合状态切换至断开状态， 模拟元器件的突然开路故障。也可以串联一个大电 阻的方式模拟元件开路。采集的信号包括输出电 压、模拟输出电压、MOS 管漏极电压、MOS 管源极电
压、二极管 $\mathrm{p}$ 极电压。采集的数据用于后续仿真 验证。

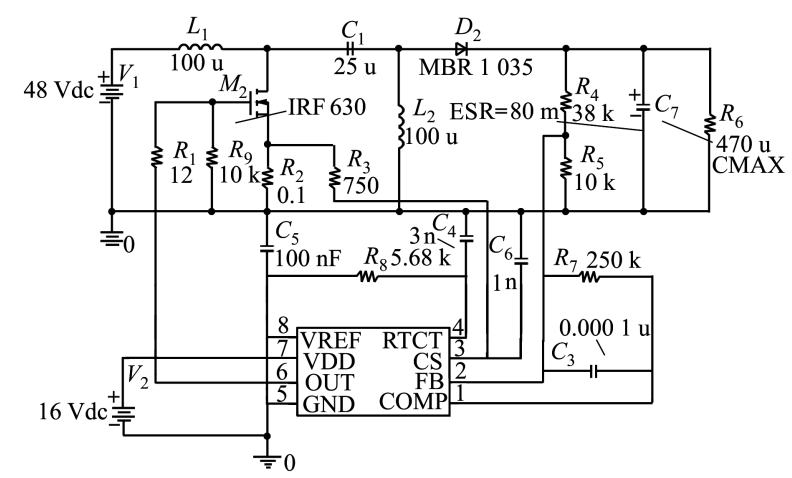

图 1 基于 Sepic 结构的 DC-DC 变换器 PSPICE 模型

\section{DC-DC 变换器故障诊断框架}

单一的模型可靠性低,而多模型融合有利于提 高故障诊断的精度和鲁棒性。本文采用的 DC-DC 变换器故障诊断技术路线如图 2 所示。主要包括数 据预处理、特征提取与选择、故障诊断建模和多模型 融合 4 个模块。数据预处理模块主要完成数据清 理、匹配和集成,便于后续对数据的分析处理; 特征 提取和优化选择模块可以将数据转化为有价值的知 识, 发现隐藏在数据集中的与故障相关的特征变量; 故障诊断建模模块通过多种智能方法建立特征变量 与故障类型之间的对应关系; 多模型融合模块通过 对多模型的优化融合提高故障诊断的精度。

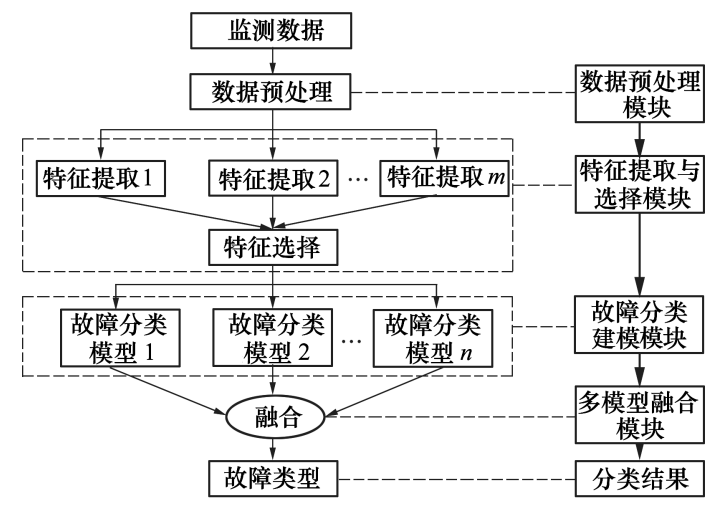

图 2 DC-DC 变换器故障分类技术路线图

\section{3 基于多模型融合的 DC-DC 变换器 实时故障诊断}




\section{1 基于神经网络的故障分类}

人工神经网络是一种基于数据的建模方法, 它 通过大量的非线性的神经元的组合和连接以及自学 习的算法,建立了输入输出之间的非线性的映射关 系。本文采用如图 3 所示的多输人多输出的 BP 神 经网络拓扑结构。

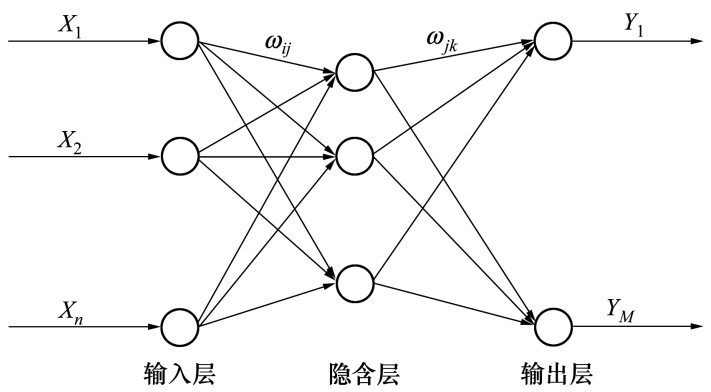

图 $3 \mathrm{BP}$ 神经网络拓扑结构图

其中, $X_{1}, \cdots, X_{n}$ 为输人变量; $Y_{1}, Y_{2}, \cdots, Y_{m}$ 是输 出变量; $\omega_{i j}$ 和 $\omega_{j k}$ 分别是网络输人层与隐含层、隐含 层与输出层神经元之间的权重。

\section{2 基于 $\mathrm{KNN}$ 的故障分类}

$K$ 最近邻 ( k-nearest neighbor, $\mathrm{KNN}$ ) 算法是一种 有效的分类算法。它以对象间的距离作为衡量指标 进行分类,一般使用欧氏距离或曼哈顿距离:

$$
\begin{aligned}
& d(x, y)=\sqrt{\sum_{k=1}^{n}\left(x_{k}-y_{k}\right)^{2}} \\
& d(x, y)=\sqrt{\sum_{k=1}^{n}\left|x_{k}-y_{k}\right|}
\end{aligned}
$$

KNN 算法描述可如下:

1) 计算测试数据与各个训练数据之间的距离;

2) 按照距离的递增关系进行排序;

3 ) 选取距离最小的 $K$ 个点;

4) 确定前 $K$ 个点所在类别的出现频率;

$5)$ 返回前 $K$ 个点中出现频率最高的类别作为 测试数据的预测分类。

\section{3 基于 PSO-SVM 的 DC-DC 变换器故障诊断 模型}

支持向量机 ( support vector machine, SVM) 具有 优异的分类性能。本部分主要针对支持向量机的训 练和优化进行阐述。选取 RBF 作为核函数, 即

$$
\kappa\left(x, x_{i}\right)=\mathrm{e}^{-\gamma\left\|x-x_{i}\right\|^{2}}
$$

式中, SVM 模型中有 2 个需要确定的参数: 惩罚因 子 $C$ 和正则化参数 $\gamma$, 采用较好的参数 $(C, \gamma)$ 能够 对故障类型有较好的分辨率。由于 SVM 采用的是
多分类,合适的结构参数选取较为困难,故本文采用 标准粒子群算法对结构参数进行寻优。基于 PSOSVM 的故障诊断流程图 4 如下:

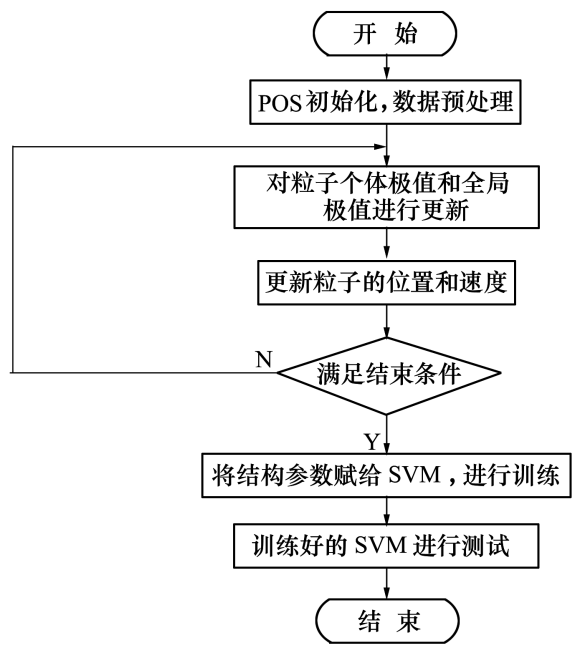

图 4 PSO 优化 SVM 结构参数流程图

\section{4 多模型融合}

多预测模型融合可用如下加权求和公式表示

$$
L=\sum_{j=1}^{M} w_{j} L_{j}(y, x)
$$

式中: $w_{j}$ 表示第 $j$ 个模型的权重系数; $y$ 表示测试数 据; $x$ 表示训练数据; $M$ 为模型的总数; $L_{j}(y, x)$ 表示 第 $J$ 个模型的分类值。可以采用加权平均法对多分 类模型的结果进行融合，即 $w_{j}=1 / M_{\circ}$ 对于结构复 杂、较难分类的数据, 分类模型的可靠性越高, 其权 重应当越大, 因此 $w_{j}$ 可定义为

$$
w_{j}=\frac{\sum_{m=1}^{M} R_{m, j} I_{\left\{\hat{f}_{j}(x) \in \Psi_{m}\right\}}}{\sum_{m=1}^{M} \sum_{j=1}^{3} R_{m, j} I_{\left\{\hat{f}_{j}(x) \in \Psi_{m}\right\}}}
$$

式中, $I_{\mid \cdot\}}$ 是示性函数。当括号内的条件满足时, $I_{|\cdot|}=1$; 否则, $I_{|\cdot|}=0$ 。模型可靠性函数定义为

$$
R_{m, j}=\exp \left(-c E_{m, j}\right)
$$

式中, $c$ 是一个待优化参数。参数 $c$ 的选取需要通过 实验数据的测试, 其最优值应当使多次实验的平均 预测误差方差最小, 其初始值应当使 $R_{m, j}$ 在 $[0,1]$ 区间内较为均衡地分布。

\section{4 仿真验证}

本部分的故障诊断算法研究主要针对于 PCM 
常见的硬故障类型, 具体包括: MOS 管短路故障、二 极管开路故障、二极管短路故障、电解电容击穿故障 和电解电容开路故障共 5 种硬故障模式, 故障的设 置详见文章第 2 部分。

实验采集的信号包括输出电压、模拟输出电压、 MOS 管漏极电压、MOS 管源极电压、二极管 $\mathrm{p}$ 极电

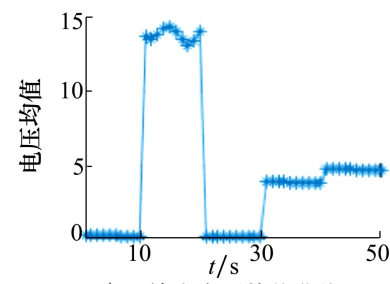

a) 输出电压均值曲线

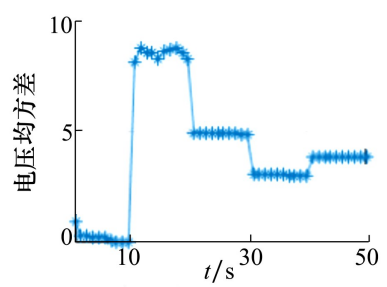

b) 输出电压标准差

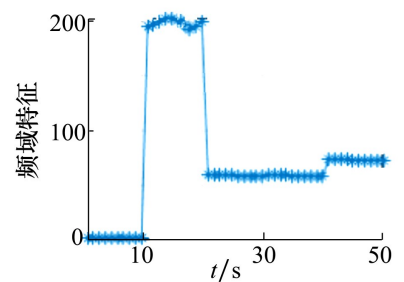

c）输出电压 FFT变换均方差曲线
压。对上述信号分别提取以下特征: (1) 时域特征变 量: 均值、均方差; 2频域特征变量: 快速傅里叶变换 (fast Fourier transform,FFT); (3)时频域特征变量: 小 波变换。

对上述采集的 5 种信号分别提取以上 4 种特 征, 部分信号统计量变化规律如图 5 所示:

图 5 输出电压信号部分统计量变化曲线

MOS 管漏极电压信号如图 6 所示:

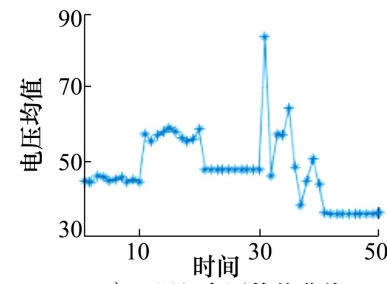

a）漏极电压均值曲线

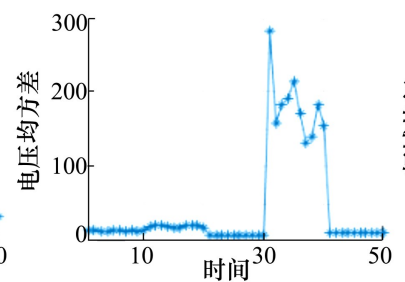

b）漏极电压标准差

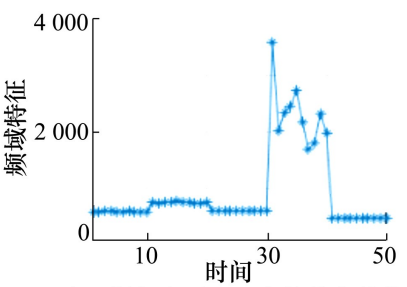

c）漏极电压 FFT 变换均方差曲线

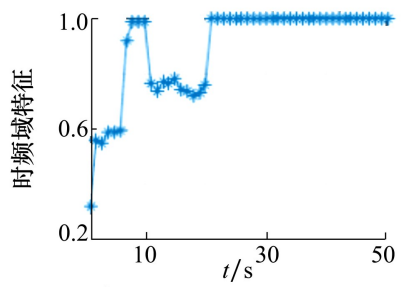

d）输出电压小波特征曲线

图 6 MOS 漏极电压信号部分统计量变化曲线

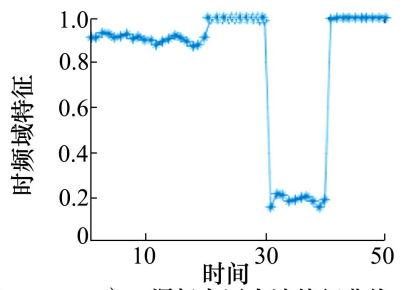

d）漏极电压小波特征曲线

二极管 $\mathrm{p}$ 极电压信号如图 7 所示:

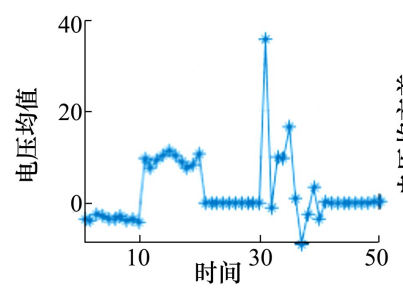

a）二极管 P 电压均值曲线

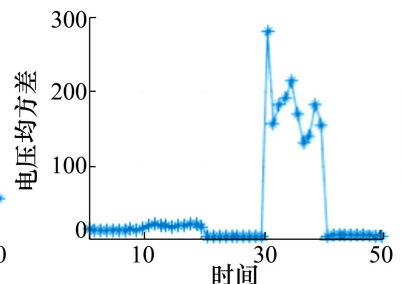

b) 二极管 P电压标准差

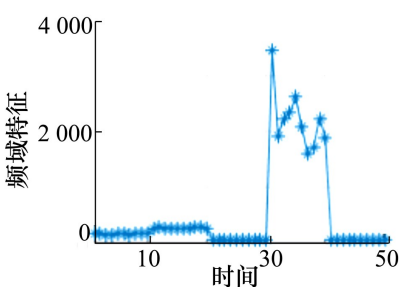

c）二极管 P变换均方差曲线

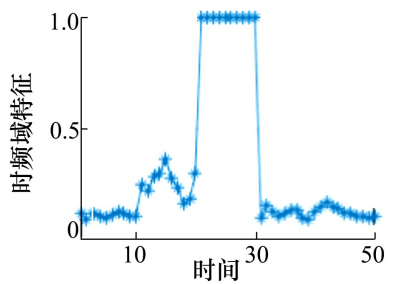

d）二极管 P 电压小波特征曲线

图 7 二极管 $\mathrm{p}$ 极电压信号部分统计量变化曲线

由于特征提取后的特征变量中包含了大量的圥 余, 对与性能退化无关的特征变量进行建模会阻碍 准确的故障诊断或使诊断的速度变慢。因此, 需要 对提取的特征进行降维。本文对上述提取后的特 征,采用主成分分析 ( principal component analysis, PCA) 进行降维处理。在降维过程中, 选取累计方差 贡献率达到 $95 \%$ 以上的前几个最大特征值对应的 特征向量作为降维后的特征向量, 主成分分析如图 8 所示, 从图中可以看出, 降维后的状态监测特征向 量维数为 4 , 大大简化了输人数据的维数, 去除了几
余,简化了模型,加快了分类速度。

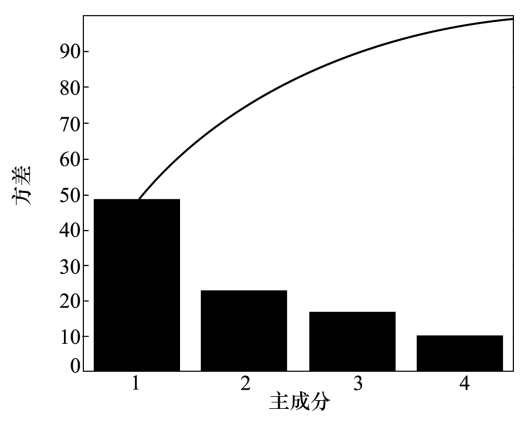

图 8 特征向量主成分分析图( $95 \%)$ 
采用 $\mathrm{BP}$ 神经网络、KNN 以及 PSO-SVM 3 种模 型的 DC-DC 变换器故障分类模型训练参数如表 1 。 仿真结果如图 9 至 11 所示。

\section{表 1 分类模型参数设置}

\begin{tabular}{|c|c|c|c|c|c|}
\hline 模型 & $\begin{array}{l}\text { 收玫 } \\
\text { 误差 }\end{array}$ & $\begin{array}{l}\text { 收敛 } \\
\text { 次数 }\end{array}$ & $K$ 值 & $\begin{array}{l}\text { 种群 } \\
\text { 规模 }\end{array}$ & $\begin{array}{l}\text { 进化 } \\
\text { 代数 }\end{array}$ \\
\hline
\end{tabular}

BP 神经网络 $0.001 \quad 4000$

KNN 5

PSO-SVM
从图中可以看出, 分类精度达到 $100 \%$ 。本文 采用加权平均法将以上 3 种模型进行优化融合, 得 到的分类结果仍然是 $100 \%$ 。仿真结果表明该多模 型融合方法对于 DC-DC 变换器的故障分类技术时 可行的。同样该模型可不失一般性的对航空电子线 路设备进行故障诊断, 通过对电路电气特性分析, 进 行特征提取和故障分类。该模型方法具有较强的适 用性和准确率。

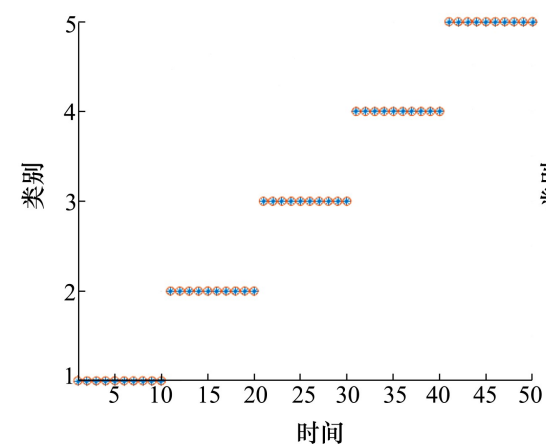

图 9 神经网络模型分类结果

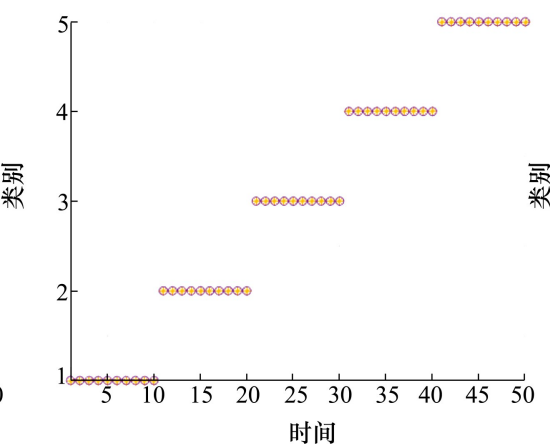

图 $10 \mathrm{KNN}$ 模型分类结果

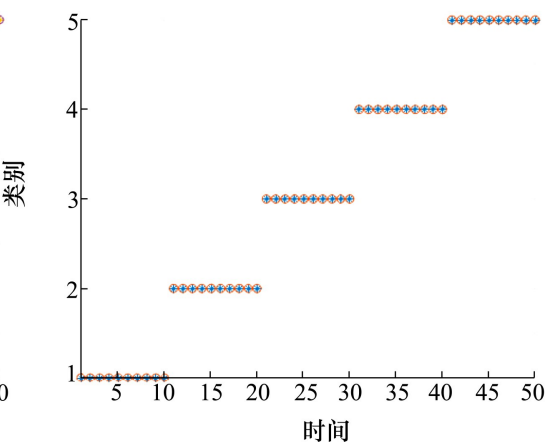

图 11 PSO-SVM 模型分类结果

\section{5 结 论}

本文首先基于 PSPICE 仿真软件, 采用 Sepic 拓 扑结构, 设计了 DC-DC 变换器模型, 并对 DC-DC 变 换器的失效规律进行了分析。对 DC-DC 变换器的 MOS 管短路故障、二极管开路故障、二极管短路故 障、电解电容击穿故障和电解电容开路故障共 5 种
硬故障模式进行模拟仿真, 并采集相关数据。对故 障数据进行了时域、频域以及时频域的特征提取, 并 进行 PCA 降维。建立了基于神经网络、KNN 以及 SVM 的多分类模型, 并将多模型分类结果进行了融 合以提高模型的鲁棒性和准确性。仿真结果表明, 本文提出的故障分类方法能有多故障进行精确的 分类。

\section{参考文献:}

［1］许明轩.飞机航电系统故障分析方法与故障诊断技术研究 $[\mathrm{J}]$. 中国设备工程, 2018, 399(14) : 102-103

XU Mingxuan. Research on fault analysis method and fault diagnosis technology of aircraft avionics system[J]. China Plant Engineering, 2018, 399(14) : 102-103 (in Chinese)

[2] PLATUS D L. Negative-stiffness-mechanism vibration isolation system[C] // Proceedings of the SPIE-the International Society for Optical Engineering, 1999: 98-105

[3] SONG Fuchao, HOU Wenkui, SHI Long. The information-enhanced fault diagnosis system design of avionics power supply module[C] // 2013 International Conference on Quality Reliebility, Rish, Maintenance, and Safety Engineering, 2013

[4] IZADIAN A, KHAYYER P. Application of Kalman filters in model-based fault diagnosis of a DC-DC boost converter[C] //36th Annual Conference on IEEE Industrial Electronics Society, Glendale, AZ, 2010: 369-372

[5] PARK T, KIM T. Novel fault tolerant power conversion system for hybrid electric vehicles[C]//2011 IEEE Vehicle Power and Propulsion Conference, Chicago, 2011 : 1-6 
[6] KIM S Y, NAM K, SONG H, et al. Fault diagnosis of a ZVS DC-DC converter based on DC-link current pulse shapes[ J]. IEEE Trans on Industrial Electronics, 2008, 55(3): 1491-1494

[7] RIBEIRO E, CARDOSO A J M, BOCCALETTI C. Fault-tolerant strategy for a photovoltaic DC-DC converter[J]. IEEE Trans on Power Electronics, 2013, 28(6): 3008-3018

[8] Sheng H, Wang F, Tipton C W. A fault detection and protection scheme for three-level DC-DC converters based on monitoring flying capacitor voltage[J]. IEEE Trans on Power Electronics, 2012, 27(2) : 685-697

\title{
Research on typical fault classification method of DC-DC converter
}

\author{
XU Zhao ${ }^{1}$, ZHANG Yitong ${ }^{1}$, PAN Zhen ${ }^{2}$, CHI Chengzhi ${ }^{2}$, LIU Xiaobin ${ }^{3}$ \\ 1.School of Electronics and Information, Northwestern Polytechnical University, Xi'an 710129, China; \\ 2.Science and Technology on Avionics Integration Laboratory, China Institute of Aeronautical Radio Electronics, \\ Shanghai 200233, China; \\ 3.Xi'an Modern Control Technology Research Institute, Xi'an 710065, China
}

\begin{abstract}
DC-DC converter is the core component of power conversion module of integrated modular avionics. Condition monitoring and fault diagnosis of DC-DC converter can effectively improve the reliability of avionics equipment, reduce the maintenance cost and greatly improve the use efficiency of aircraft. In this paper, firstly, a typical DC-DC converter model based on SEPIC topology is designed in PSPICE environment, and the failure modes of DC-DC converter are analyzed. Secondly, the typical fault types of DC-DC converter are simulated, and the corresponding original data are obtained through simulation. Finally, the processing framework including data preprocessing, feature extraction and selection, and multi model fusion is used to do fault classification of the DC-DC converter. The fault diagnosis of the converter is simulated. Simulation results show the effectiveness of the proposed method.
\end{abstract}

Keywords : DC-DC converter; failure modes; feature extraction; fault classification; multi model fusion ; simulation; SEPIC topology

引用格式: 徐㻇, 张一童, 潘震, 等. $D C-D C$ 变换器的典型故障分类方法研究 $[J]$. 西北工业大学学报, 2021,39(1): 71-76

$X U$ Zhao, ZHANG Yitong, PAN Zhen, et al. Research on typical fault classification method of DC-DC converter[J]. Journal of Northwestern Polytechnical University, 2021, 39(1): 71-76 (in Chinese) 